\title{
A Survey on Modulation Schemes Used for Link Adaptation in WiMAX Networks
}

\author{
P. Vinoth \\ Assistant Professor, Dept of ECE, \\ Ganadipathy Tulsi's Jain Engineering College, \\ Vellore, TamilNadu, India
}

\author{
P. Jayakumar \\ Assistant Professor, Dept of CSE, \\ Arunai Engineering College, Thiruvannamalai, \\ TamilNadu, India
}

\begin{abstract}
Worldwide Interoperability for Microwave Access (WiMAX) is a telecommunications technology that uses radio spectrum to transmit bandwidth between digital devices. WiMAX has the ability to transmit large amount of data to large distance. The IEEE standard for WiMAX defines an adaptive modulation framework which allows a WiMAX system to communicate with various burst profiles according to the link quality. This adaptive modulation framework is also called as link adaptation. This link adaptation scheme uses different modulation schemes for different communication link quality. This paper analysis different modulations schemes used in WiMAX link adaptation.
\end{abstract}

\section{Keywords}

WiMAX, link adaptation, QPSK, 16QAM, 64QAM, BPSK

\section{INTRODUCTION}

The IEEE 802.16 standard is more popularly known as Worldwide Interoperability for Micro Wave Access (WiMAX). WiMAX network is a wireless Metropolitan Area Network provides network access to subscribers through exterior antennas communicating with central radio base stations (BSs). The wireless Metropolitan Area Network offers an alternative to cabled access networks, such as fiber optic links, coaxial systems using cable modems, and digital subscriber line (DSL) links. The MAC Layer of IEEE 802.16 was designed for PMP broadband wireless access applications. It is designed to meet the requirements of very high data rate applications with a variety of quality of service (QoS) requirements. The signaling and bandwidth allocation algorithms have been designed to accommodate hundreds of terminals per channel [1].

Link adaptation is a way to increase data rates in wireless systems by adapting transmission parameters such as the modulation and coding rate [2]. Link adaptation has an important role in broadband wireless communication systems. Traditionally, link adaptation refers to the concept of adjusting transmit parameters like the modulation order and coding rate dynamically according to channel conditions. When the channel is good, higher-order modulation and higher code rates are chosen, while when the channel is bad, less efficient lowerorder modulation and lower code rates are used. The wireless channel condition is constantly changing in time and frequency. Link adaptation is a technique used to improve spectrum efficiency by adapting system parameters, such as modulation scheme and coding rate, according to the channel conditions [3]. Mobile WiMAX networks achieve high spectral efficiency using adaptive modulation and coding schemes [9].

Digital modulation scheme converts the digital information signal into the signal which can be transferred to the communication channel. There are two main types of digital modulation. First type will keep the amplitude as constant and variable frequency or phase of the carrier. Examples are FSK and PSK. Where as in second type keeps frequency and phase as constant and varying amplitude based on information. Example is ASK modulation. These different types of digital modulation schemes help in implementing link adaptation in WiMAX network. Some of the important digital modulation schemes are FSK, MSK, BPSK, QPSK and QAM modulation. Frequency-shift keying (FSK) is a digital frequency modulation. In which frequency of the carrier is varied based on binary information signal. One of the basic FSK scheme is Binary Frequency Shift Keying (BFSK). In BFSK two frequency levels are used for modulation. They are logic 1 and logic 0 . Phase Shift Keying (PSK) also a digital modulation scheme. Here phase of the carrier signal is varied based on the digital information. One of the basic PSK modulations is Minimum Shift Keying (MSK). It is used in many applications. One of the variants of MSK is Gaussian filtered Minimum Shift Keying (GMSK). It is used as a modulation scheme in the GSM cellular telecommunication system. This MSL scheme has few more additional advantages over other schemes. So it is widely used in communication system. Minimum shift keying (MSK) is a form of phase shift keying (PSK) that is used in a number of applications. A variant of MSK modulation, known as Gaussian filtered Minimum Shift Keying, GMSK, is used for a number of radio communications applications including being used in the GSM cellular telecommunications system. In addition to this MSK has advantages over other forms of PSK and as a result it is used in a number of radio communications systems. Another commonly used PSK scheme is Binary Phase Shift Keying (BPSK). It is also used in a variety of communication system. This modulation uses two different phase value which is separated by $180^{\circ}$. This modulation is also called as 2-PSK Compared with other PSK modulations, this modulation is most robust. It will be more convenient to predict the symbol in the high noisy environment also. But it has a drawback of low data rate. It can module at one bit per symbol (shown in the figure). So this modulation scheme is not suitable for high data-rate applications with limited bandwidth. The Quadrature Phase Shift keying (QPSK) is another PSK modulation scheme. It is also termed as Quaternary PSK, 4-PSK and quadriphase PSK. This modulation scheme uses 4-points on the constellation diagram. So it can module at a rate 2 bits/symbol. The next important modulation scheme is Quadrature amplitude modulation (QAM). This modulation scheme combines ASK and PSK. It can provide high data rate. So it is widely used in many digital communication systems.

In IEEE802.16 based WiMAX network, only QPSK and QAM modulations only used [4]. To implement link adaptation in WiMAX network, modulation scheme of base station modified to one of this digital modulation schemes. When the signal to noise ratio is high QAM modulation is used and when the signal to noise ratio is low QPSK modulation is used. This paper analysis modulation schemes used with link adaptation of 
WiMAX network and suggest a new modulation scheme to include in link adaptation. In [10] Li Chen, Ying Yang, Xiaohui Chen, and Guo Wei proposed an Energy efficient link adaptation scheme, but they concentrated on QAM modulation only. In [11] Riccardo Andreotti, Ivan Stupia, Vincenzo Lottici, Filippo Giannetti, and Luc Vandendorpe proposed link adaptation scheme. They used multi level QAM modulation scheme

The remainder of this paper is organized as follows: In Section II, describe about WiMAX networks. In Section III, describe about Link Adaptation. In Section IV, describe about digital modulation and explained different digital modulation schemes. In Section V, describe about modulation scheme used in WiMAX Link Adaptation. In Section VI, propose a modulation scheme to include in existing WiMAX link adaptation. . Section VII contains some concluding remarks.

\section{WIMAX NETWORKS}

IEEE 802.16 family of standards is officially called WirelessMAN in IEEE, it has been commercialized under the name "WiMAX" (from "Worldwide Interoperability for Microwave Access") by the industry alliance called the WiMAX Forum. The name "WiMAX" was created by the WiMAX Forum, which was formed in June 2001 to promote conformity and interoperability of the standard. The mission of the Forum is to promote and certify compatibility and interoperability of broadband wireless products based on the IEEE 802.16 standards. WiMAX is a standards-based technology enabling the delivery of last mile wireless broadband access as an alternative to cable and DSL. The current WiMAX revision provides up to $40 \mathrm{Mbit} / \mathrm{s}$ with the IEEE $802.16 \mathrm{~m}$ update expected to offer up to $1 \mathrm{Gbit} / \mathrm{s}$ fixed speeds. The most popular implementation of the IEEE 802.16 standard is the Mobile WirelessMAN originally defined by the 802.16e-2005 amendment.

The early iterations of IEEE 802.16 focused on line-of-sight (LOS) applications using high-frequency bands between 10 to $66 \mathrm{GHz}$. More recently, efforts have been focused on specifying amendments to earlier standards to support non-line-of-sight (NLOS) applications between 2 to $11 \mathrm{GHz}$. The 802.16-2004 standard, more commonly known as $802.16 \mathrm{~d}$, was published in 2004. [5] The 802.16e standard is an amendment to the $802.16 \mathrm{~d}$ standard and was ratified at the end of 2005 and published as 802.16-2005. While the 802.16d standard supports fixed and nomadic applications, the 802.16e standard supports fixed, nomadic, portable and mobile solutions [6].

\section{LiNK ADAPTATION}

The principle of Link Adaptation is simple, when the radio link is good, use a high-level modulation; when the radio link is bad, and use a low-level, but also robust, modulation. Link adaptation plays a central role in regulating the utilization of the radio resources [7]. There are three possible physical approaches to adapt to the varying link condition:

- Power adaptation: Higher transmit power could improve data reception at the receiver. However, high transmit power may lead to interference to other communication systems nearby when the network topology is considered. Algorithms in this category concern the best utilization of radio resources by regulating the transmit power at the transmitter. Higher power may be allocated for transmission under poor link condition.
- $\quad$ Frequencies adaptation: Noise and signal fading to the link is frequency-selective. Some wireless networks such as the IEEE 802.11a WLAN standard adopts the frequency-hopping spread spectrum mechanism. Algorithms in this category such as use this method to switch between different frequencies ranges.

- Modulation rate adaptation: Modulation coding schemes (MCSs) perform differently under various link conditions. For example, the binary phase shift keying (BPSK) works well in poor link condition while 64quadrature amplitude modulation (64QAM) does not. 64QAM provides higher bandwidth efficiency than BPSK and it results in higher throughput for the system. When the link condition is poor, bandwidth efficiency is sacrificed for robust communication between the transmitter and the receiver.

Different MCSs and message formats are specified in the WiMAX standard for the systems to deliver broadband service. However, the policy on how and which modulation scheme should be used under various link conditions are not specified. There is a wide research space to develop and evaluate different link adaptation algorithms for enhancing the performance of the WiMAX systems.

\section{Digital Modulation Schemes}

Digital modulation is a method of decoding information for transmission. Information or a voice conversation is turned into a series of digital bits; the $0 \mathrm{~s}$ and $1 \mathrm{~s}$ of computer binary language. At the receiving end, the information is reconverted. The past few years have seen a major transition from the simple amplitude modulation (AM) and frequency modulation (FM) to digital techniques such as Quadrate Phase Shift Keying (QPSK), Frequency Shift Keying (FSK), Minimum Shift Keying (MSK) and Quadrate Amplitude Modulation (QAM).

Digital terrestrial microwave radio designers, concentrate on bandwidth efficiency and lower error rate. But they do not worry about power efficiency because they have plenty of power available. Whereas handheld cellular phone designers, concentrate on power efficiency because all the handheld cellular phones are battery running devices. So power efficiency plays an important role. They concentrate on cost efficiency also. They want to produce a cell phone at the cheapest cost. If a developer concentrates on improvement of one parameter (power, cost and bandwidth), developer have to sacrifice other parameter. The variation of the property of a signal, such as its amplitude, frequency or phase is called modulation. This process carries a digital signal or message. Different types of modulation techniques are available such as, Amplitude Shift Keying (ASK), Frequency Shift Keying (FSK) and Phase Shift Keying (PSK).

There are variety of digital modulation scheme are used in telecommunication system. These varieties of modulation scheme are obtained by varying one of characteristics of the carrier signal like amplitude, frequency, phase or a combination of these characteristics are:

\subsection{Amplitude-shift keying(ASK)}

Amplitude-shift keying (ASK) is a form of digital modulation scheme. Here amplitude of a carrier wave is varied based on the digital modulating signal. And here frequency and phase of the signal are kept constant. And in this method to represent the logic 0 , one type of amplitude is used. And to represent the logic 1, another type of amplitude is used. This 
will be considered on and off of the carrier signal. So this modulation scheme also termed as ON/OFF keying.

\subsection{Minimum shift keying (MSK)}

Minimum shift keying (MSK) is a special type of continuous phase-frequency shift keying (CPFSK) with $\mathrm{h}=0.5$. A modulation index of 0.5 corresponds to the minimum frequency spacing that allows two FSK signals to be coherently orthogonal, and the name minimum shift keying implies the minimum frequency separation (i.e. bandwidth) that allows orthogonal detection.

\subsection{Phase shift keying (PSK)}

Phase shift keying (PSK) is widely used in a variety of radio communication systems. It is well suited for large coverage areas. PSK, phase shift keying enables data to be carried on a radio communications signal in a more efficient manner than Frequency Shift Keying, FSK, and some other forms of modulation. Phase of the carrier signal is a digital modulation scheme which conveys data by modulating or changing of carrier waves. The most commonly and widely used are Binary Phase shift Keying (BPSK) and Quadrature Phase Shift Keying (QPSK). Other PSKs are Differential Phase Shift Keying (DPSK) and Multilevel Phase Shift Keying (MPSK) etc.

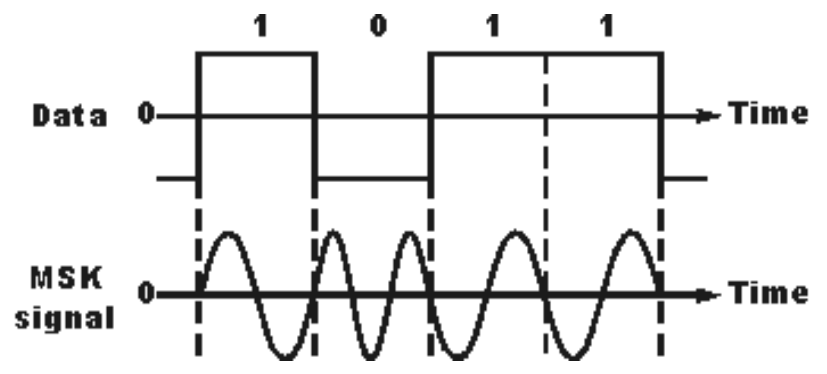

Fig 1: Signal using MSK modulation

\subsection{Binary Phase Shift Keying (BPSK)}

Like any form of shift keying, there are defining states or points that are used for signaling the data bits. One of the basic PSK schemes is binary phase shift keying (BPSK). It is also termed as Phase Reversal Keying (PRK). A digital signal alternating between +1 and -1 (or 1 and 0 ) will create phase reversals, i.e. 180 degree phase shifts as the data shifts state. This is also known as two-level PSK as it uses two phases separated by $180^{\circ}$ to represent binary digits. The principle equation is,

$S(t)=A \cos (2 \pi f c t)$ for binary $1, A \cos (2 \pi f c t+\pi)$ for binary 0 .

$S(t)=A \cos (2 \pi f c t)$ for binary $1,-A \cos (2 \pi f c t)$ for binary 0 .

This kind of phase modulation is very effective and robust against noises especially in low data rate applications as it can modulate only 1 bit per symbol.

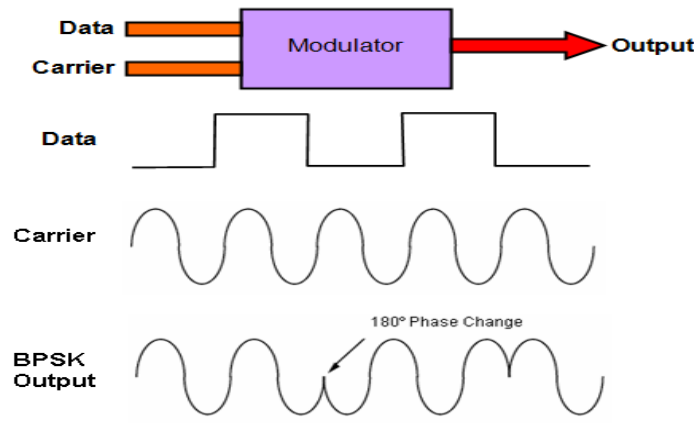

(a)

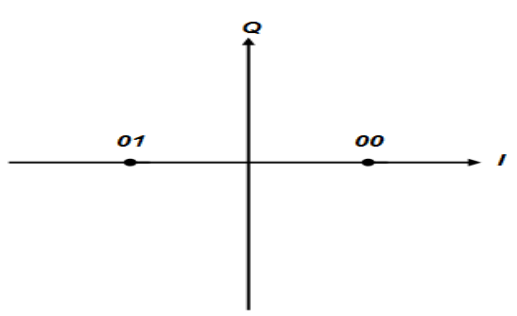

(b)

Fig 2: BPSK, (a) Block Diagram (b) Constellation

\subsection{Quadrature Phase shift keying (QPSK)}

This is also known as four-level PSK where each element represents more than one bit. Each symbol contains two bits and it uses the phase shift of $\pi / 2$, means $90^{\circ}$ instead of shifting the phase $180^{\circ}$. The principle equation of the technique is:

$S(t)=A \cos (2 \pi f c t+\pi / 4)$ for $11, A \cos (2 \pi f c t+3 \pi / 4)$ for 01 ,

$\mathrm{A} \cos (2 \pi \mathrm{fct}-3 \pi / 4)$ for 00 and $\mathrm{A} \cos (2 \pi \mathrm{fct}-\pi / 4)$ for 10 .

In this mechanism, the constellation consists of four points but the decision is always made in two bits. This mechanism can ensure the efficient use of bandwidth and higher spectral efficiency.

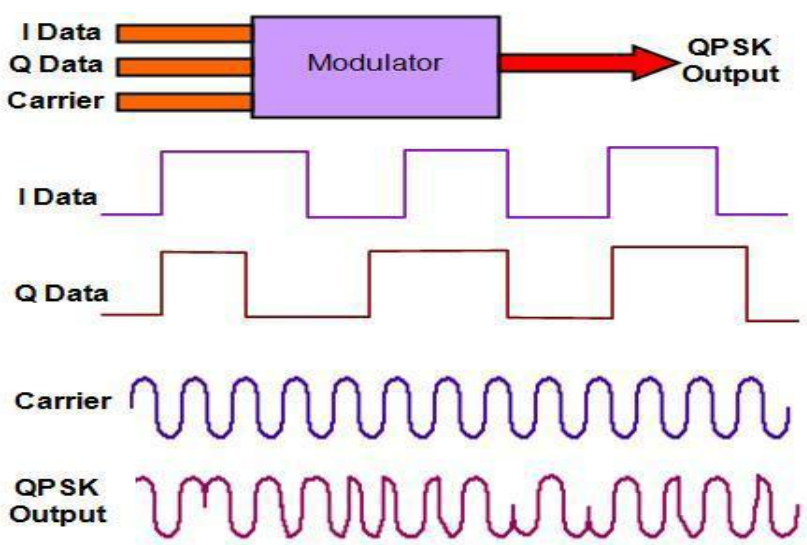




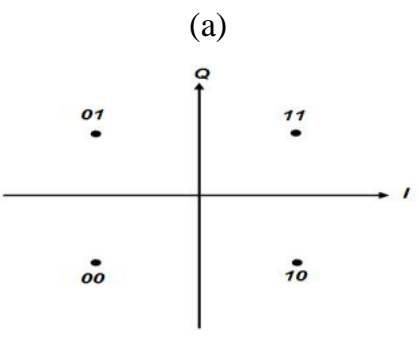

(b)

Fig 3: QPSK, (a) Block Diagram (b) Constellation

\subsection{Quadrature amplitude modulation (QAM)}

Quadrature amplitude modulation is mostly used in data communications applications and digital radio communication systems. There are so many types of QAM modulation scheme are available. Few types are 16 QAM, 32 QAM, 64 QAM, 128 QAM, and 256 QAM. The various flavors of QAM may be used when data-rates beyond those offered by 8-PSK are required by a radio communications system. The QAM modulation scheme has an advantage of high data rate. It can module high number of bits per symbol compared with other modulation scheme. When modulation order increases the number of bits encoded in the symbol will also increase. So when higher order QAM is selected, the data rate of the channel can be increased. The principle equation is:

$S(t)=d 1(t) \cos 2 \pi f c t+d 2(t) \sin 2 \pi f c t$

\subsection{6-states Quadrature Amplitude Modulation (16QAM)}

This is called 16-states Quadrature Amplitude Modulation which means four different amplitude levels would be used and the combined stream would be one of $16=4 * 4$ states. In this mechanism, each symbol represents 4 bits.

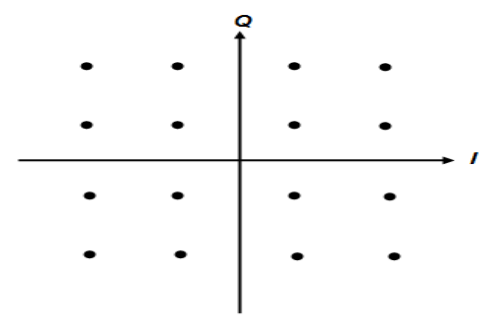

Fig 4: 16 QAM Constellation

\subsection{2-states Quadrature Amplitude Modulation (32QAM)}

This is called 32-states Quadrature Amplitude Modulation which means five different amplitude levels would be used. In this mechanism, each symbol represents 5 bits.

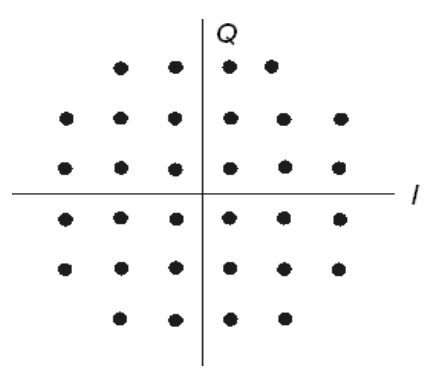

Fig 5: 32 QAM Constellation

\subsection{4-states Quadrature Amplitude Modulation (64QAM)}

This is same as 16 QAM except it has 64-states where each symbol represents six bits $(26=64)$. It is a complex modulation techniques but with greater efficiency. The total bandwidth increases according to the increasing number of states for each symbol. Mobile WiMAX uses this higher modulation technique when the link condition is high.

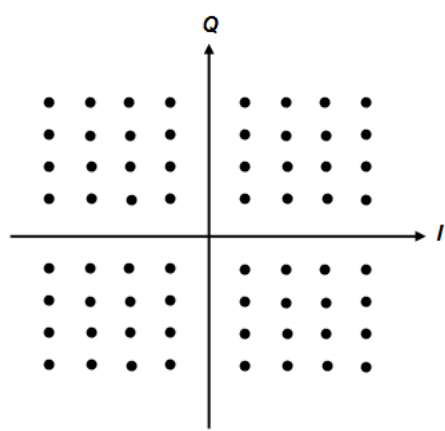

Fig 6: 64 QAM Constellation

\section{Modulation Schemes USEd In WiMAX NETWORKS}

In WiMAX network Link adaptation scheme, only three modulation schemes are used as given in IEEE standard [6] When a channel is good, i.e., with high SNR value 64QAM modulation is used, when a channel is average, 16QAM is used and when channel is bad QPSK is used.

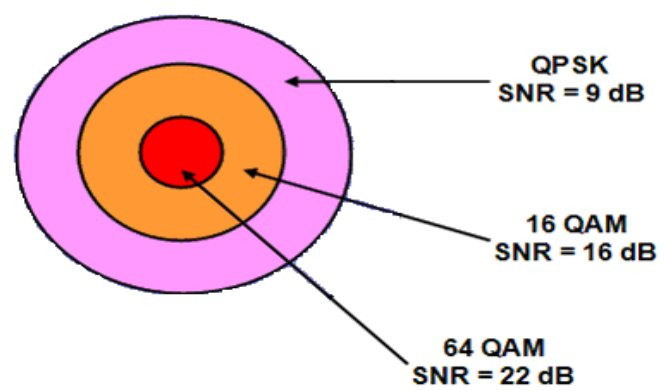

Fig 7: Adaptive Modulation Scheme of WiMAX 
When WiMAX Subscriber Station (SS) has SNR in the rage of $22 \mathrm{~dB}$ then 64QAM modulation scheme will be used that SS. When WiMAX SS has SNR in the rage of $16 \mathrm{~dB}$ then 16QAM modulation scheme will be used that SS. When WiMAX SS has SNR in rage of $9 \mathrm{~dB}$ then a QPSK modulation scheme will be used that SS.

Modulation and coding schemes for WiMAX network is given in the table. The different coding rate will be used to achieve different bit per symbol rate. As given in table, when a developer uses 64QAM developer can achieve high peak data rate as compared with other modulation scheme. QPSK achieves a lower peak data rate. QPSK allows OFDM symbol to carry less information bits, but 64QAM allows OFDM symbol to carry more information bits compared with QPSK [8]. The drawback of 64QAM is it will produce more noise when the channel is bad, so when channel is good 64QAM is used. When a channel is bad QPSK modulation scheme is used, since it will work properly with noisy channel.

Table 1: Modulation and coding schemes for WiMAX

\begin{tabular}{|c|c|c|c|c|c|}
\hline $\begin{array}{c}\mathbf{R} \\
\mathbf{A} \\
\mathbf{T} \\
\mathbf{E} \\
\mathbf{I D}\end{array}$ & $\begin{array}{c}\text { Modulation } \\
\text { rate }\end{array}$ & Coding & $\begin{array}{l}\text { Information } \\
\text { bits/symbol }\end{array}$ & $\begin{array}{c}\text { Information } \\
\text { bits/ } \\
\text { OFDM } \\
\text { symbol }\end{array}$ & $\begin{array}{c}\text { Peak } \\
\text { data } \\
\text { rate in } \\
5 \\
\text { MHz } \\
(\mathrm{Mb} / \mathrm{s})\end{array}$ \\
\hline 1 & QPSK & $1 / 2$ & 1 & 184 & 3.95 \\
\hline 2 & QPSK & $3 / 4$ & 1.5 & 280 & 6.00 \\
\hline 3 & 16QAM & $1 / 2$ & 2 & 376 & 8.06 \\
\hline 4 & 16QAM & $3 / 4$ & 3 & 568 & 12.18 \\
\hline 5 & 64QAM & $2 / 3$ & 4 & 760 & 16.30 \\
\hline 6 & 64QAM & $3 / 4$ & 4.5 & 856 & 18.36 \\
\hline
\end{tabular}

\section{Proposed Modulation Scheme For WIMAX NETWORKS}

In existing adaptive modulation scheme of WiMAX, QPSK, 16QAM and 64QAM modulation scheme are used as per IEEE standard. The developer suggests a BPSK modulation scheme also can be included in an existing adaptive modulation scheme to improve its stability.

BPSK scheme can work in the high noisy channel as compared with QPSK. So it will allow Base Station (BS) to transmit data to SS when the channel condition is too bad. It will improve the quality of service with little drop in overall data rate. Inclusion of BPSK scheme will reduce the overall data rate of network but it will improve stability of the network, which will work properly with the high noisy channel.

BPSK modulation scheme with a coding rate of $1 / 2$ will give 0.5 information bits/symbol. Then an OFDM symbol can transmit 88 information bits. Peak data rate in $5 \mathrm{MHz}$ will be 1.89

Inclusion of the BPSK modulation scheme in adaptive modulation scheme will increase its layer count from 3 to 4 . The BPSK modulation scheme will form the outer layer. So, as per new adaptive modulation scheme for WiMAX networks, when SNR of channel is $22 \mathrm{~dB}$ range then 64QAM modulation scheme will be used, when SNR of channel is $16 \mathrm{~dB}$ range then 16QAM modulation scheme will be used, when SNR of channel is 9dB range then the QPSK modulation scheme will be used, when SNR of channel is $6 \mathrm{~dB}$ range then the BPSK modulation scheme will be used.

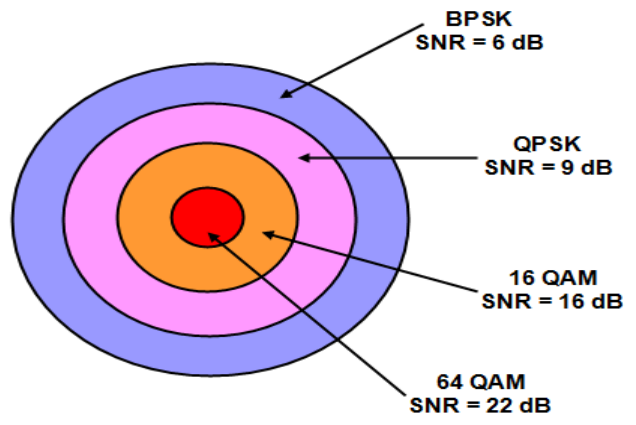

Fig 8: New Adaptive Modulation Scheme for WiMAX

\section{CONCLUSION}

This paper analyzed various modulation schemes used in WiMAX link adaptation. QPSK, 16QAM and 64QAM modulation schemes are used in WiMAX network to implement link adaptation. When the SNR of the channel is high QAM modulation scheme is used, when SNR of the channel is low QPSK modulation is used. In addition to these modulation schemes, the paper proposes a BPSK modulation scheme has to be included in an adaptive modulation framework to support very low SNR value also. The addition of the BPSK modulation scheme will improve the reliability of link adaptation.

\section{REFERENCES}

[1] C. Eklund, R. B. Marks, K. L. Stanwood, and S. Wang, "IEEE standard 802.16: A technical overview of the Wireless MAN air interface for broadband wireless access," IEEE Commun. Mag., vol. 40, no. 6, pp. 98-107, Jun. 2002

[2] T.H.Chan, M.Hamdi,C.Y.Cheung and M.Ma, "A link adaptation algorithm in mimo based wimax systems", Journal of Comm., vol 2, no. 5, pp.16-24, Aug. 2007

[3] Chan-Byoung Chae; Forenza.A.; Heath.R.W.; McKay.M.; Collings.I, "Adaptive MIMO transmission techniques for broadband wireless communication systems ",Communications Magazine IEEE, vol 48, no. 5, pp. 112 118, May 2010

[4] Chakchai So-In, Raj Jain and Abdel-Karim Tamimi, "Capacity Evaluation for IEEE 802.16e Mobile WiMAX", Journal of CSC, Networking, and Comm.,, vol. 1, no. 1, April 2010

[5] R.-T. Juang, P. Ting, H.-P. Lin and D.-B. Lin, "Link adaptation based on repetition coding for mobile worldwide interoperability for microwave access systems", IET Commun., 2010, Vol. 4, Iss. 9, pp. 1039-1048, Sep. 2009

[6] IEEE Standard for Local and Metropolitan Area Networks-Part 16: Air Interface for Fixed Broadband Wireless Access Systems, IEEE Std. 802.16-2004, 2004

[7] IEEE Standard for Local and Metropolitan Area Networks-Part 16: Air Interface for Fixed and Mobile Broadband Wireless Access Systems, IEEE Std. 802.16e2005, 2005

[8] A. Ghosh, D. R.Wolter, J. G. Andrews, and R. Chen, "Broadband wireless access with WiMax/802.16: Current performance benchmarks and future potential," IEEE Commun. Mag., vol. 43, no. 2, pp. 129-136, Feb. 2005

[9] Seung-Eun Hong and Meejoung Kim, "Design and Analysis of a Wireless Switched Digital Video Scheme for Mobile TV Services over WiMAX Networks" IEEE TRANSACTIONS ON BROADCASTING, vol. 59, no. 2, June 2013 
[10] Li Chen, Ying Yang, Xiaohui Chen, and Guo Wei, "Energy-Efficient Link Adaptation on Rayleigh Fading Channel for OSTBC MIMO System With Imperfect CSIT", IEEE TRANSACTIONS ON VEHICULAR TECHNOLOGY, vol. 62, no. 4, May 2013
[11] Riccardo Andreotti, Ivan Stupia, Vincenzo Lottici, Filippo Giannetti, and Luc Vandendorpe, "Goodput-Based Link Resource Adaptation for Reliable Packet Transmissions in BIC-OFDM Cognitive Radio Networks", IEEE TRANSACTIONS ON SIGNAL PROCESSING, vol. 61, NO. 9, May 1, 2013 